OPEN

SUBJECT AREAS:

TOUCH RECEPTORS

PATTERN VISION

Received

24 October 2013

Accepted

20 November 2013

Published

13 December 2013

Correspondence and requests for materials should be addressed to M.I. (ide@rikkyo.ac.jp) or S.H. (hidaka@ rikkyo.ac.jp)

\section{Tactile stimulation can suppress visual perception}

\author{
Masakazu Ide \& Souta Hidaka
}

Department of Psychology, Rikkyo University, 1-2-26, Kitano, Niiza-shi, Saitama, 352-8558 Japan.

An input (e.g., airplane takeoff sound) to a sensory modality can suppress the percept of another input (e.g., talking voices of neighbors) of the same modality. This perceptual suppression effect is evidence that neural responses to different inputs closely interact with each other in the brain. While recent studies suggest that close interactions also occur across sensory modalities, crossmodal perceptual suppression effect has not yet been reported. Here, we demonstrate that tactile stimulation can suppress the percept of visual stimuli: Visual orientation discrimination performance was degraded when a tactile vibration was applied to the observer's index finger of hands. We also demonstrated that this tactile suppression effect on visual perception occurred primarily when the tactile and visual information were spatially and temporally consistent. The current findings would indicate that neural signals could closely and directly interact with each other, sufficient to induce the perceptual suppression effect, even across sensory modalities.

eople continuously receive large amounts of sensory inputs from the surrounding environment. Our brain automatically and efficiently integrates these inputs to be perceptually organized. In addition to additive/ facilitatory processes, suppressive/inhibitory processes should exist in order to constantly receive new incoming information and update internal representations. In fact, the percept of an input (e.g., talking voices of neighbors) can be easily suppressed by another input (e.g., airplane takeoff sounds). This perceptual suppression effect has been investigated within single modalities (e.g., vision ${ }^{1-3}$ ). A neurophysiological study ${ }^{4}$ reported that masking stimuli, whose onsets were temporally preceded or succeeded by that of a target, suppressed neural responses related to the target onset or offset signal, respectively. This indicates that the perceptual suppression effect occurs due to direct interactions between neural responses to different inputs in the brain.

The brain receives and integrates information across sensory modalities in order to establish coherent and robust percepts $s^{5}$. For example, perceived intensity of a visual stimulus can be enhanced by a concurrent auditory stimulus $^{6}$ at neural level interactions ${ }^{7}$. Studies on crossmodal interactions have mainly demonstrated such additive/facilitatory effects ${ }^{8,9}$, and assume that each bit of sensory information is independently sent from primary sensory areas to some higher sensory association areas to be integrated ${ }^{10}$. A recent study ${ }^{11}$, however, suggests that crossmodal inputs can induce activations in the cortex, which has been considered a sensory specific area, indicating that direct interactions could occur between primary sensory cortices. Moreover, it has been reported that there exist multimodal neurons (e.g., visuo-tactile neurons) that have very close or overlapping neural receptive fields for stimuli in different sensory modalities ${ }^{12}$. It is therefore very likely that the perceptual suppression effect can also occur across sensory modalities.

Here, we demonstrate that tactile input can suppress the percept of visual stimuli. In these experiments, tactile stimulation (vibration) was applied to the observer's index finger on the palm side of their hand, which was placed forward and covered by a black cloth. We presented Gabor patches as visual target stimuli on a display in front of the observers (Fig. 1A). A simple detection task (e.g., reporting whether a target is presented or not) may involve a response/decisional bias (e.g., tactile stimulation induce frequent visual present or absent responses/judgments) instead of a perceptual effect. Thus, we introduced visual orientation discrimination as an indirect perceptual task; observers judged whether stripes of the visual stimuli were perceived as tilting left or right. We found that tactile stimulation degraded visual orientation discrimination performance. Moreover, this effect frequently occurred when tactile and visual stimuli were presented in a spatially and temporally consistent manner. These results reveal that tactile stimulation can induce the perceptual suppression effect on visual perception.

\section{Results}

Suppression effect of tactile stimulation on visual discrimination. In Exp. 1, we presented Gabor patches (0.5 $\times 0.5 \mathrm{deg}, 5.88 \mathrm{cycle} / \mathrm{deg}, \sigma=1.30 \mathrm{deg}, 180 \mathrm{deg}$ of phase angle) as target stimuli against a gray background 


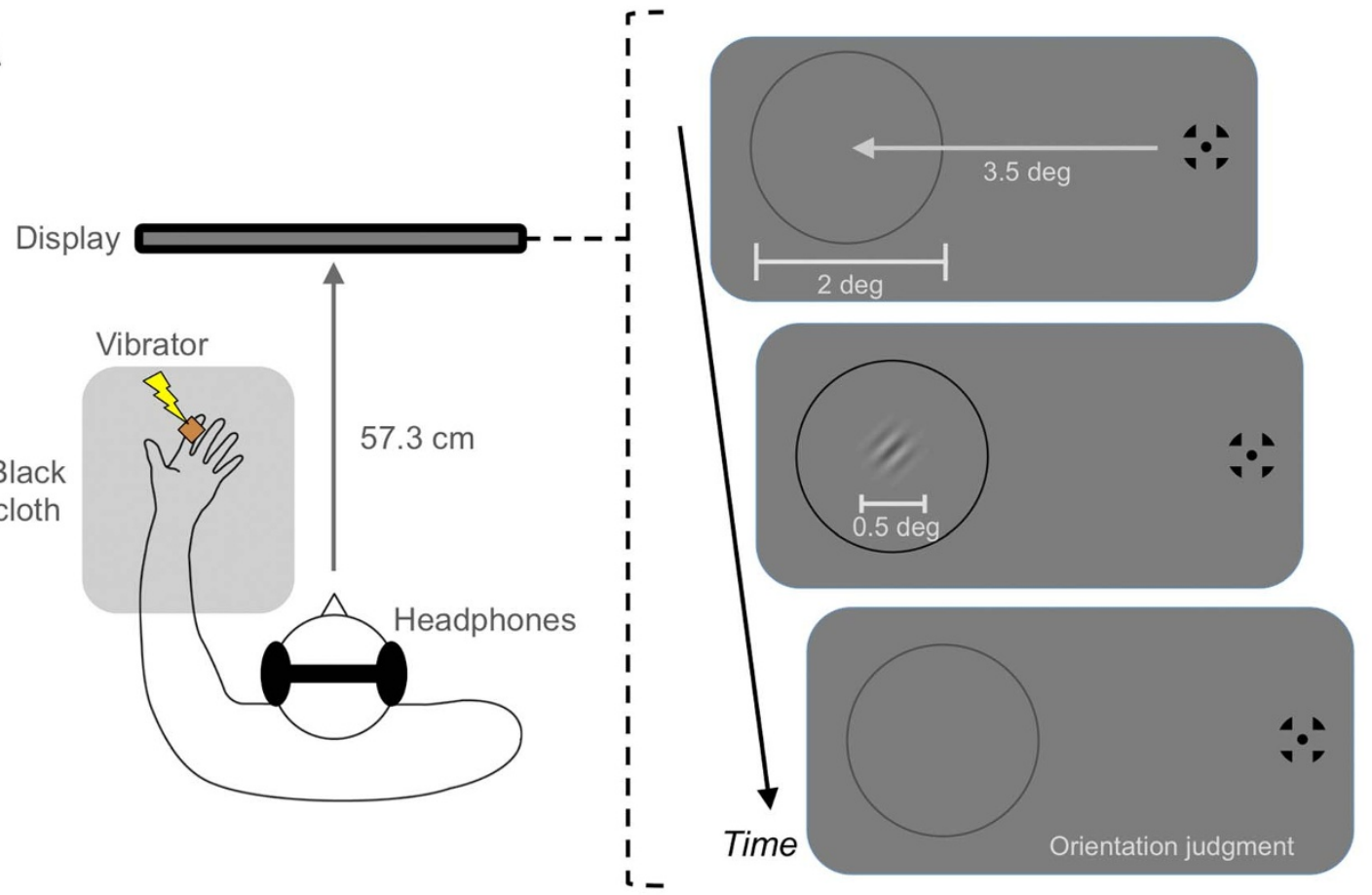

B
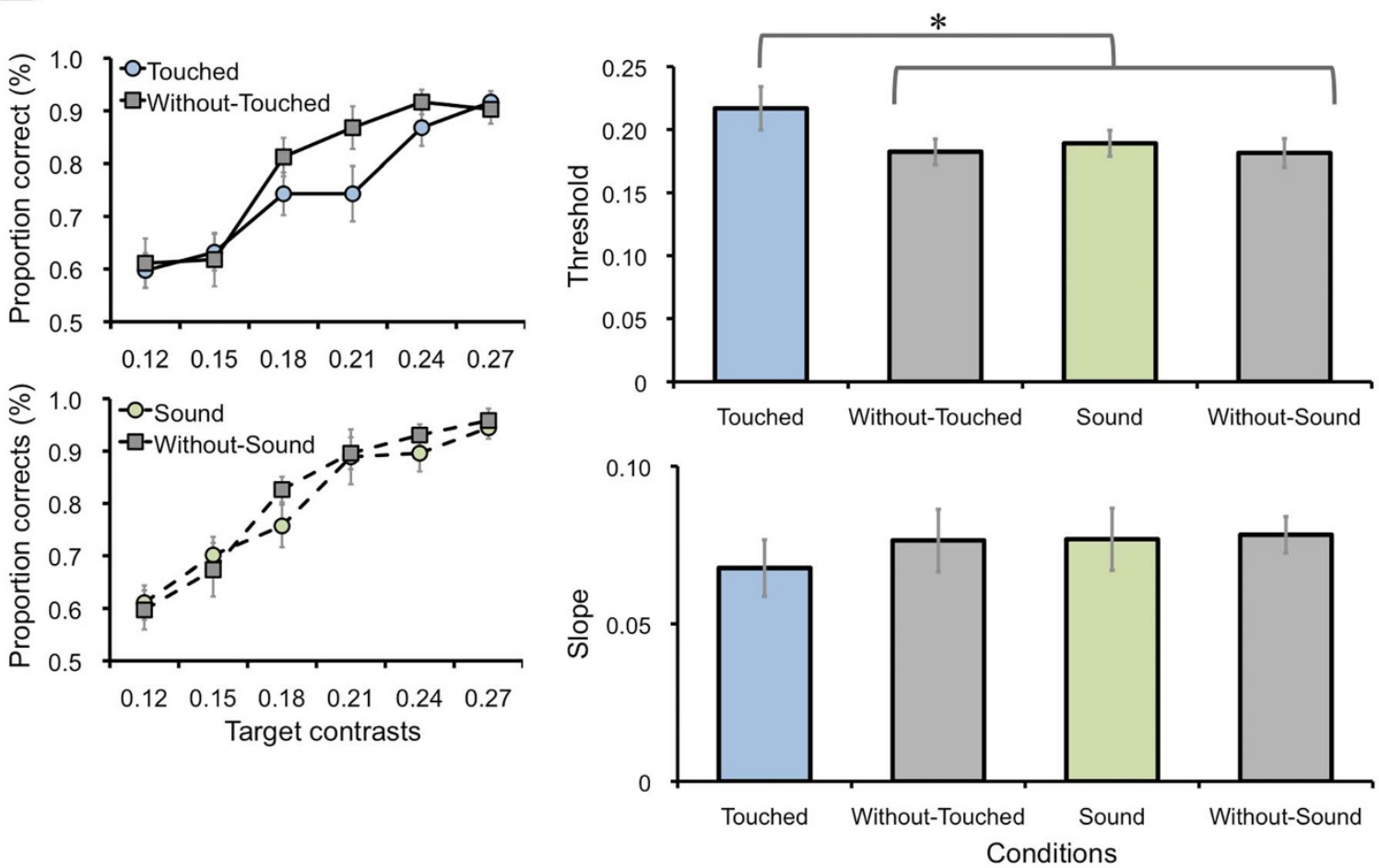

Figure 1 Suppression effect of tactile stimulation on the visual orientation discrimination task (Exp. 1). (A) Schematic illustrations of tactile and visual stimuli. A vibrator was set on the participant's left index finger of hand, which was covered by a black cloth. After presentation of a fixation point $(0.6 \times$ $0.6 \mathrm{deg}$ ) and a gray ring ( $2 \mathrm{deg}$ in diameter, $0.05 \mathrm{deg}$ in width) for 1200-2000 ms (randomly assigned in each trial), a visual target $(0.5 \times 0.5 \mathrm{deg})$ tilting either left or right was presented at the center of the ring for $20 \mathrm{~ms}$. The target's contrast was either $0.12,0.15,0.18,0.21,0.24$, or 0.27 . The ring color was also changed from gray to black as the cue for the target onset. Tactile stimulation was concurrently applied for $200 \mathrm{~ms}$. White noise bursts were also delivered through headphones to mask sounds emitted by the vibrator. After presentation of the target, participants were asked to judge the target's orientation. (B) Psychometric functions. Proportions of correct responses for visual discrimination were plotted against the target contrasts. The point of $75 \%$ responses of the functions (thresholds) and slopes were estimated. (C) Threshold and slopes. Threshold was increased when the tactile stimulation was applied (Touched condition) relative to when the sound (Sound condition) or no stimulus was presented (Without-Touched and Without-Sound conditions). These tendencies were not observed for the slopes. Error bars denote the standard error of the mean $(\mathrm{N}=8)$. An asterisk indicates the condition was significantly different from the others $(p<.05)$. Illustrations produced by and used with permission from Shoko Yabuki. 
$\left(30.41 \mathrm{~cd} / \mathrm{m}^{2}\right)$ for $20 \mathrm{~ms}$. We asked 8 participants to judge whether the orientation of the target was perceived as tilting left ( $-45 \mathrm{deg})$ or right (+45 deg) (Fig. 1A). The target's contrast (Weber contrast) was either $0.12,0.15,0.18,0.21,0.24$, or 0.27 . During the presentation of the target, vibration ( $460 \mathrm{~Hz}$ sinusoidal burst) was applied to the participant's left index finger for $200 \mathrm{~ms}$ as the tactile stimulus (Touched condition). Sounds emitted by the vibration were masked by white noise bursts delivered through headphones (Fig. 1A). Trials without the tactile stimulus were also included as a baseline (Without-Touched condition). We plotted proportions of correct responses against the target contrasts (Fig. 1B). This revealed that the proportions of the Touched condition were lower than that of the Without-Touched condition. We also tested whether sounds, a sensory input other than tactile stimulation, could also be effective on visual discrimination. White noise bursts $(200 \mathrm{~ms})$, instead of vibration, were delivered thorough speakers (covered by a black cloth) placed around the participant's index finger (Sound condition). The perceived intensity of the sound was adjusted by the participants as subjectively consistent with that of the tactile stimulus (67.63 (average) \pm 6.20 (standard deviation) dBA). Trials without the auditory stimulus were also introduced as a baseline (Without-Sound condition). The proportions of correct responses in Sound and Without-Sound conditions were almost identical (Fig. 1B). We estimated the $75 \%$ point of the proportions as threshold by linear regression analysis in each condition for each participant (Fig. 1C). A one-way repeated measures of analysis of variance (ANOVA) revealed a main effect of the conditions $[F(3,21)$ $=5.17, p<.01]$. A post hoc test (Tukey's HSD) revealed that the threshold of the Touched condition was larger than the other conditions $(p<.05)$. These tendencies were not observed for the slopes of the functions, which is considered an index of sensitivity $[F(3,21)=0.38, p=.77]$. These results thus indicate that a tactile stimulus can suppress the percept of visual stimuli. Absence of the auditory effect also indicates that this suppression effect on visual perception was primarily observed for tactile stimulation, suggesting that the effect could not be simply explained by spatial attentional capture from the visual stimuli to the other sensory inputs ${ }^{13}$.

Spatial aspects of the tactile suppression effect. Exp. 2 investigated spatial aspects of the tactile suppression effect on visual perception. We stimulated the index finger, forearm, or chest on the left side of the body for 9 participants (Index finger, Forearm, and Chest conditions) (Fig. 2A). These body parts were assumed to have similar tactile sensitivities ${ }^{14}$. The Without-Touched condition was also included as a baseline. The visual targets with 0.21 of contrast level were presented on either the left or right visual field, so that they were congruent or incongruent with the tactile stimulus in space, respectively. Target orientation discrimination performance was again degraded by tactile stimulation to the index finger when the visual and tactile stimuli were at the ipsilateral, congruent position (Fig. 2B). Regarding the Index finger condition, a two-way repeated measures ANOVA with the tactile (Touched/Without-Touched) and spatial (Congruent/Incongruent) conditions revealed a significant interaction effect between these factors $[F(1,8)=6.53$, $p<.05]$. A simple main effect of tactile condition was significant in the Congruent condition $[F(1,16)=13.22, p<.005]$, but not in the Incongruent condition $[F(1,16)=0.03, p=.87]$. These tendencies were not observed for the Forearm and Chest conditions; the corresponding interaction effects were not significant in both conditions $[F s(1,8)=1.28,1.46$, $p s=.29, .26$, respectively]. These results indicate that the tactile suppression effect was observed specifically when the tactile stimulus was applied to the index finger, and when the visual and tactile stimuli were congruent in space.

Exp. 3 further investigated spatial congruency aspects of the tactile suppression effect on visual perception. The tactile stimulus was applied to the left or right index finger of each 8 participant, and the visual target was presented in the left or right visual field for each half of the participants, respectively. We asked the participants to uncross or cross their hands. In the Hands-Uncrossed condition, the left visual target and the left hand tactile stimulus were congruent both in space and body coordinates. In the Hands-Crossed condition, however, the left visual target was spatially congruent with the right hand tactile stimulus. In contrast, the left visual target was consistent with the left hand tactile stimulus in body coordinates (Fig. 3A). The Without-Touched condition was also included as a baseline. We found that the tactile stimulus degraded discrimination performance for visual target orientation, when the tactile and visual stimuli were presented at the ipsilateral, congruent position in space, regardless of whether hands were crossed or uncrossed. A three-way repeated measures ANOVA with hand (Hands-Uncrossed/Crossed), tactile (Touched/Without-Touched), and spatial (Congruent/Incongruent) conditions found a significant interaction effect between the latter two factors $[F(1,7)=11.12, p<.05]$. A significant simple main effect of tactile condition in the Congruent condition $[F(1,14)=$ $6.07, p<.05$ ] revealed that the proportion correct in the Touched condition was lower than that of the Without-Touched condition, while the effect in the Incongruent condition $[F(1,14)=4.76, p<$ .05] showed the opposite tendency. Therefore, the tactile suppression effect was observed especially when the visual and tactile stimuli were presented in a spatially congruent manner.

Temporal aspects of the tactile suppression effect. Exp. 4 investigated temporal aspects of the tactile suppression effect on visual perception. Since the tactile suppression effect could occur when the visual and tactile stimuli were temporally overlapped, we manipulated the inter-stimulus interval (ISI) between these stimuli from $-250 \mathrm{~ms}$ (tactile stimulus first) to $+250 \mathrm{~ms}$ (visual stimulus first) in 50-ms steps for 10 participants (Fig. 4A). We also included the Without-Touched condition as a baseline. We estimated the amount of tactile effect by subtracting the proportions of correct responses in the Without-Touched condition from those in each ISI condition for each participant (Fig. 4B). Larger values indicate a greater tactile effect. A one-way repeated measures ANOVA revealed a significant main effect of ISI $[F(8,72)=2.16, p<.05]$. We estimated the peak values of the ISI functions by fitting a quadratic function to each participant's data using a least squares method. The average peak is $53.85 \mathrm{~ms}$ and the $95 \%$ confidence interval is from $-15.49 \mathrm{~ms}$ to $123.19 \mathrm{~ms}$. These results indicate that the tactile suppression effect occurs when visual and tactile stimuli are presented within a certain temporal range.

\section{Discussion}

The present study demonstrates that tactile stimulation applied to the index finger of an observer's hand degrades visual orientation discrimination performance. This tactile suppression effect on visual perception was not observed when the tactile stimulation was applied to the forearm or chest. We also showed that the tactile suppression effect occurred when the tactile and visual stimuli were presented at ipsilateral, congruent positions in space, regardless of whether hands were crossed or uncrossed. Furthermore, the tactile suppression effect primarily occurred when the tactile and visual stimuli were presented within a certain temporal range. These results reveal that a tactile stimulus to the hand can induce the suppression effect on visual perception when these stimuli are spatially and temporally consistent.

A study reported that tactile stimulation increased the detection threshold for sounds ${ }^{15}$. In this study, tactile stimulation was transiently presented during a sequence of sounds varying in intensity. Thus, attentional capture from a sound to tactile stimulus would be considered a key factor for this tactile effect ${ }^{16}$. Similar crossmodal attentional effects have been reported as attentional blink ${ }^{17}$ or change 
A

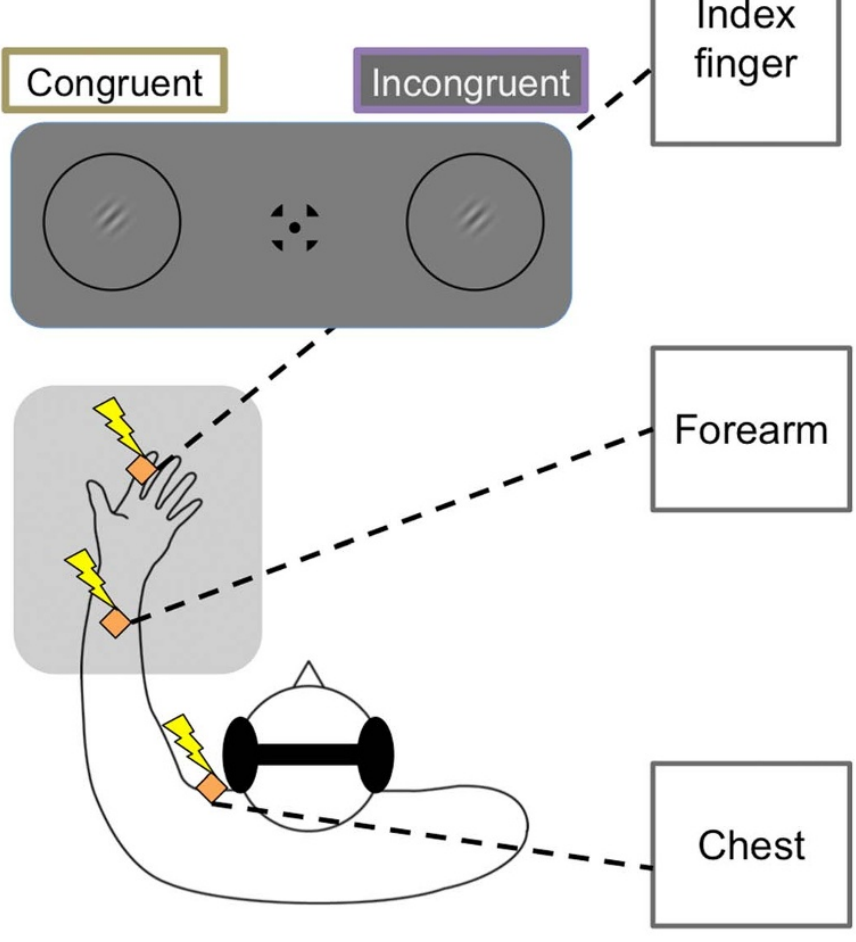

ฮั

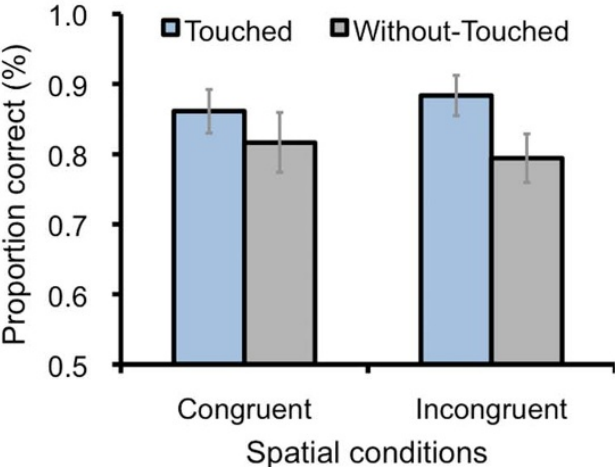

Figure 2 Effects of body parts and spatial congruency (Exp. 2). (A) Schematic illustrations of visual and tactile conditions. Tactile stimulation was applied to the index finger, forearm, or chest on the left side of participants' bodies. The visual target ( 0.21 of contrast level) was presented on either the left or the right visual field, so that it was congruent or incongruent with the tactile stimulus in space, respectively. (B) Results. Proportions of correct responses for visual discrimination were degraded only when the tactile stimulus was applied to the index finger and when the visual and tactile stimuli were at the congruent position in space. Error bars denote the standard error of the mean $(\mathrm{N}=8)$. An asterisk indicates the condition was significantly different from the others $(p<.05)$. Illustrations produced by and used with permission from Shoko Yabuki.

blindness ${ }^{18}$. However, these crossmodal attentional effects could not fully explain our current findings. The degradation of visual discrimination performance was not observed when the sounds were presented around the participants' index fingers, although both tactile and auditory stimuli could equally capture the participants' attention to the stimulus locations ${ }^{13}$. We also found that the tactile suppression effect on visual perception primarily occurred when the visual and tactile stimuli were presented within a temporal window that peaked around $50 \mathrm{~ms}$ of ISI (i.e., visual target first). This characteristic is incompatible with the crossmodal attentional effect because attentional cueing stimuli were reported to be effective when they temporally preceded target stimuli ${ }^{19,20}$. Rather, the tactile enhancement effect, instead of the interference effect, was observed when the tactile stimulus temporally preceded the visual stimulus $(-200 \mathrm{~ms}$ and $150 \mathrm{~ms}$ of ISI in Exp. 4) as well as when they were presented in a spatially incongruent manner (the Incongruent condition in Exp. 3). One might also assume the involvement of response/decisional biases. However, the spatial and temporal congruency aspects of the tactile suppression effect on visual perception, as well as the adoption of the indirect perceptual task (visual orientation discrimination), could exclude the interpretations based on these biases. We could thus consider that the tactile suppression effect could occur at a perceptual processing stage.

Crossmodal researches have demonstrated interactions between visual and tactile information ${ }^{21}$, specifically as additive/facilitatory effects from vision to tactile sensation. For instance, the sight of body parts, such as the hand or forearm, could alter or improve tactile sensitivity ${ }^{22,23}$ or enhance visuo-tactile interaction ${ }^{24}$. Neurophysiological studies have also reported that macaque premotor cortical areas responded not only to tactile stimulation but also to visual hand images $^{25}$. Similarly, human brain-imaging studies have found that activations in the somatosensory cortex, such as the parietal and premotor cortical regions, were modulated by not only tactile information but also by visual information ${ }^{26}$. Further, the sight of hands could modulate neural activation to tactile inputs in the somatosensory cortical regions ${ }^{27,28}$. The results of the current study could add two new findings regarding visuo-tactile interactions: the suppressive/inhibitory effect can occur from tactile sensation to vision.

Neurophysiological evidence has suggested that perceptual suppression effect in a single modality (vision) could occur due to direct 

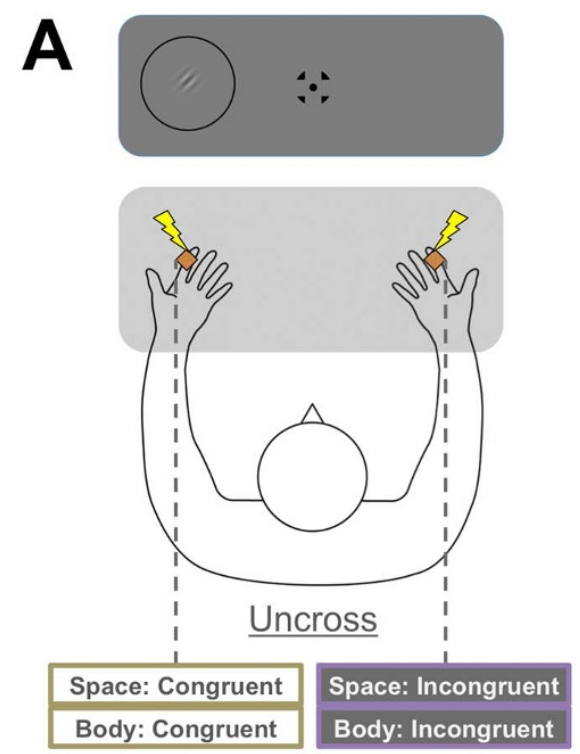
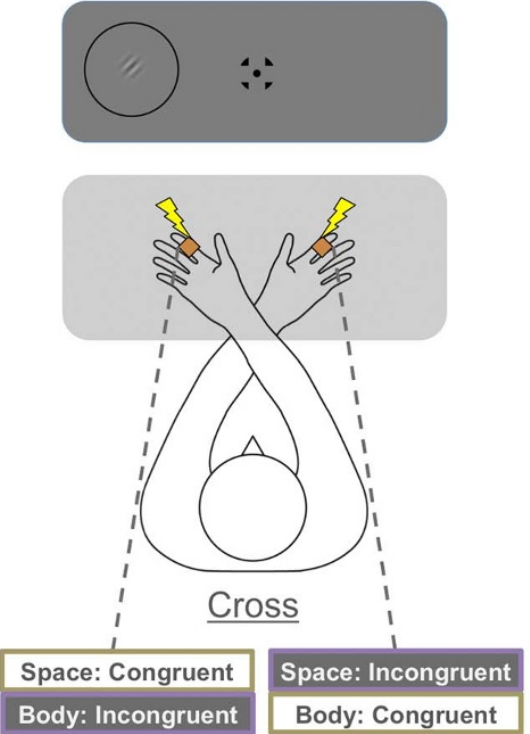

B

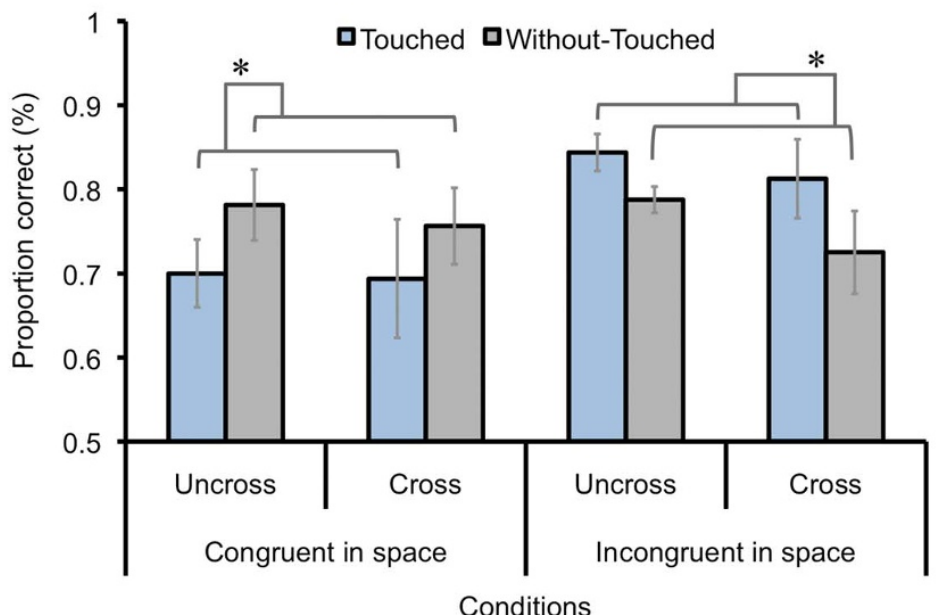

Figure $3 \mid$ Effects of spatial congruency with or without hands-crossed (Exp. 3). (A) Schematic illustrations of visual and tactile conditions. While the visual target was presented on either the left or the right visual fields, the tactile stimulus was applied to either the left or the right index finger. Participants' hands were uncrossed or crossed. In the Hands-Uncrossed condition, the left visual target and the left hand tactile stimulus were congruent both in space and body coordinates. In the Hands-Crossed condition, whereas the left visual target was spatially congruent with the right hand tactile stimulus, the left visual target was consistent with the left hand tactile stimulus in body coordinates. (B) Results. Proportions of correct responses for visual discrimination were degraded only when the tactile and visual stimuli were congruent in spatial coordinates. Error bars denote the standard error of the mean $(\mathrm{N}=8)$. Asterisks indicate the conditions with a significant difference $(p<.05)$. Illustrations produced by and used with permission from Shoko Yabuki.

interaction/suppression of neural responses to different stimuli ${ }^{4}$. Studies on crossmodal interaction have also suggested that there exist direct interactions among primary sensory cortices, including vision and tactile sensation ${ }^{11,29}$. It has also been reported that visuo-tactile bimodal neurons in the ventral intraparietal (VIP) area in macaque monkeys have firmly congruent receptive fields for each type of stimuli $^{30,31}$. Based on these findings, the tactile suppression effect on visual perception could also occur by direct interaction of neural responses to visual and tactile inputs.

We found that the tactile suppression effect on visual perception primarily occurred when the tactile stimulus was applied to the index finger of hand, which was covered by a black cloth. It was reported that visuo-tactile bimodal neurons in somatosensory cortices, such as the VIP area, have visual receptive fields surrounding areas of the hand $^{32}$. Moreover, this type of receptive field could be also mapped onto a displayed visual hand image when the visual image and tactile/ proprioceptive sensations of a covered hand are associated based on their spatiotemporal consistency ${ }^{33}$. These findings would suggest that tactile stimulation and related neural responses could be associated with the visual target on the display in our experimental situation. It would also be noteworthy that the peak of the temporal window for the tactile suppression effect on visual perception was estimated to shift toward tactile stimulus delayed timing (around $50 \mathrm{~ms}$ of ISI). These characteristics are compatible with visual metacontrast masking ${ }^{1,34}$; a target visual stimulus is masked by a following surrounding masker, which is assumed to trigger excitatory feedback and lateral inhibition from the masker to the $\operatorname{target}^{35}$ (Fig. 5A). We demonstrated that the visual perceptual suppression effect, comparable with the tactile suppression effect, could be induced by a visual masker with $0.55 \mathrm{deg}$ of width and 0.37 of contrast (Weber contrast) at $30 \mathrm{~ms}$ and $80 \mathrm{~ms}$ of ISI relative to the target (Fig. 5B). This result would suggest that tactile stimulation could be represented, and 
A
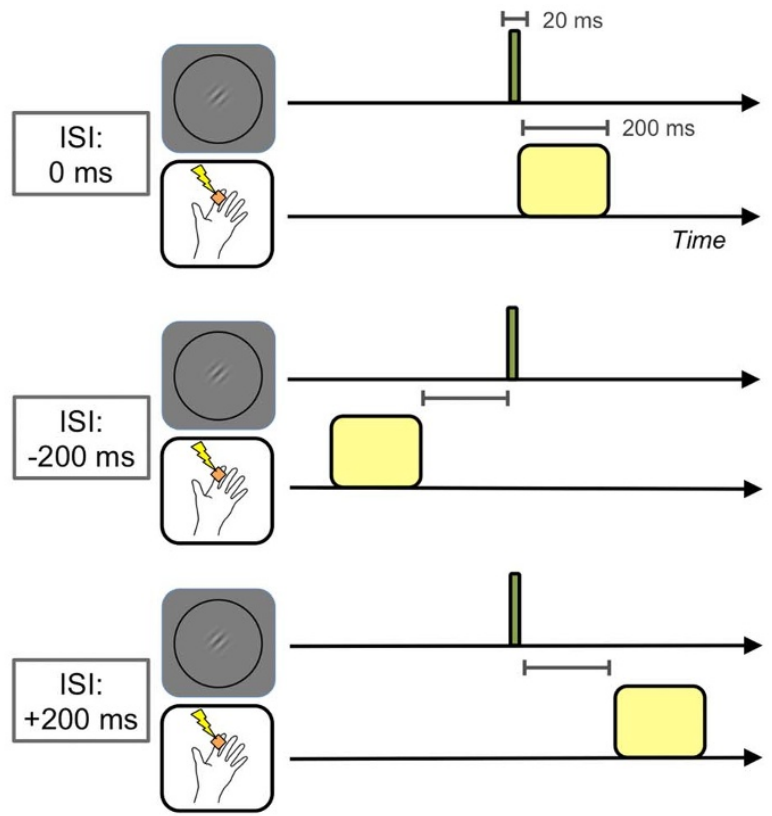

B

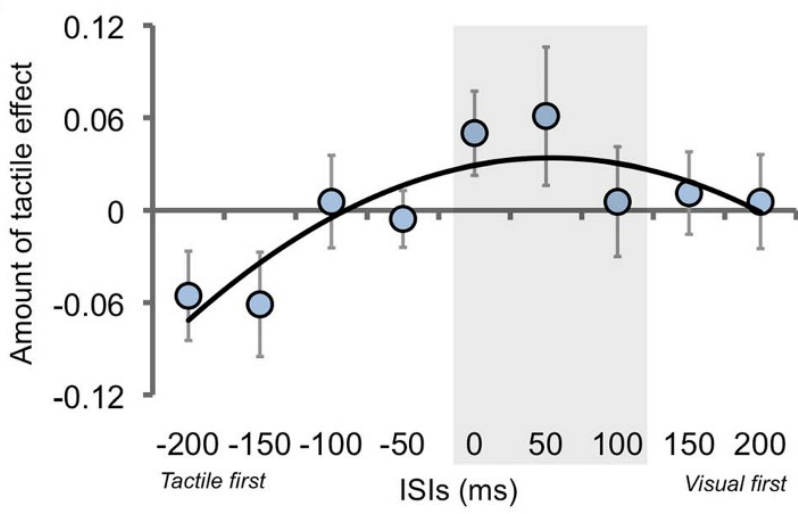

Figure $4 \mid$ Effects of temporal distance between visual and tactile stimuli (Exp. 4). (A) Schematic illustrations of the inter-stimulus interval (ISI) between the visual and tactile stimuli. ISIs were assigned from $-250 \mathrm{~ms}$ (tactile stimulus first) to $+250 \mathrm{~ms}$ (visual stimulus first) in 50-ms steps. (B) Results. After calculating the amount of tactile effect in each ISI based on proportions of correct responses for visual discrimination, we estimated peak values of ISI functions in each participant's data using a least squares method. The average peak is $53.85 \mathrm{~ms}$ and the $95 \%$ confidence interval ranges from -15.49 ms to 123.19 ms (gray shaded area). A black curved line indicates a fitted quadratic function to the averaged data $\left(\mathrm{R}^{2}=.74\right)$. Illustrations produced by and used with permission from Shoko Yabuki.

could function as this visual masker in the tactile suppression effect on visual perception. The underlying mechanisms of the tactile suppression effect on visual perception, including whether and how primary visual and somatosensory cortices interact with each other through visuo-tactile bimodal neurons and/or direct feedforward and feed-back neuronal connections, should be firmly investigated in near future with neurophysiological and brain imaging techniques.

\section{Methods}

Participants and apparatus. Each of the participants $(8,9,8$, and 10 participants in Exp. 1, 2, 3, and 4, respectively) had normal or corrected-to-normal vision and normal hearing. The participants were naive to the purpose of the experiment. Informed consent was obtained from each participant before conducting the experiments. All procedures were approved by the local ethics committee of Rikkyo University. Visual stimuli were presented on a linearized CRT display (EIZO, FlexScan T566, 17 inch) with a resolution of $1024 \times 768$ pixels and a refresh rate of $100 \mathrm{~Hz}$. The viewing distance was $57.3 \mathrm{~cm}$. A black cloth covered the participant's hand and forearm. Tactile stimuli were presented through an audio interface (Roland, EDIROL FA-66) and a vibrator (Eishindenki, Attachable Speaker M-PZT-02). In order to mask the sounds emitted by the vibrator, white noise bursts ( $56.1 \mathrm{dBA}$ ) were generated digitally (sampling frequency $44.1 \mathrm{kHz}$ ) and delivered through headphones (Sennheiser HDA200). In Exp. 1, auditory stimuli (white noise bursts) were also delivered through the audio interface and speakers (Dell, AX210). A customized PC (Dell Precision T5500 workstation) and MATLAB (MathWorks, Inc.) with the Psychophysics Toolbox ${ }^{36,37}$ were used to control the experiment. A numeric keypad and a foot switch (FS3-P, Route-R) was used for recording responses in Exps.1-3 and Exp. 4, respectively. We confirmed that the onset of the visual and tactile or auditory stimuli was synchronized using a digital oscilloscope (OWON, PDS5022TFT). The observers were instructed to place their heads on a chin rest. All experiments were conducted in a dark room.

Stimuli. For visual stimuli, a fixation point consisting of bull's eye and cross hair (0.6 $\left.\times 0.6 \mathrm{deg} ; 0.52 \mathrm{~cd} / \mathrm{m}^{2}\right)^{38}$ and a gray ring (2 deg in diameter, $0.05 \mathrm{deg}$ in width; $\left.15.04 \mathrm{~cd} / \mathrm{m}^{2}\right)$ was presented on a gray background $\left(30.41 \mathrm{~cd} / \mathrm{m}^{2}\right)$. The ring was presented at the left (Exps. 1, 4) or at either the left or right (Exps. 2, 3) position relative to the fixation point in $3.5 \mathrm{deg}$ of horizontal distance. We also presented a Gabor patch $(0.5 \times 0.5 \mathrm{deg}, 5.88 \mathrm{cycle} / \mathrm{deg}, \sigma=1.30 \mathrm{deg}, 180 \mathrm{deg}$ of phase angle $)$ as a target for $20 \mathrm{~ms}$. The stripes of the target stimulus tilted either left ( $-45 \mathrm{deg}$ ) or right (+45 deg). The target's contrast (Weber contrast) was either $0.12,0.15,0.18,0.21$, 0.24 , or 0.27 in Exp. 1, and was fixed at 0.21 in the other experiments. The ring color was changed from gray to black $\left(0.52 \mathrm{~cd} / \mathrm{m}^{2}\right)$ during target presentation. As a tactile stimulus, a vibration (460- $\mathrm{Hz}$ sinusoidal burst) was applied for $200 \mathrm{~ms}$ with $1 \mathrm{~ms}$ of cosine ramp at the onset and offset. The vibrator was attached to the participant's index finger on the palm side of their hand in Exps. 1 and 4, to the left index finger/left forearm/left side of the chest in Exp. 2, and to the left/right index fingers in Exp. 3. In Exp. 1, auditory stimuli (white noise bursts) were also delivered through two speakers placed around the participant's index finger for $200 \mathrm{~ms}$ with $1 \mathrm{~ms}$ of cosine ramp at the onset and offset. The perceived intensity of the auditory stimuli was adjusted by the participants as subjectively consistent with that of the tactile stimulus (67.63 (average) \pm 6.20 (standard deviation) $\mathrm{dBA}$ ).

Procedure. In Exp. 1, after presentation of the fixation point and the gray ring for 1200-2000 ms (randomly assigned in each trial), the visual target was presented. The ring color also changed from gray to black as the cue of target onset. Concurrently, the tactile (Touched condition) or the auditory (Sound condition) stimulus was presented. The situation without the tactile (Without-Touched condition) or the auditory (Without-Sound condition) stimulus was also included. The tactile and auditory trials were introduced as a blocked design. After the target presentation, the gray ring reappeared and the participants were asked to judge whether the target was perceived as tilting left or right. This experiment consisted of training and main sessions. The training session consisted of 72 trials without presentation of the tactile or auditory stimulus: Target's contrasts $(6) \times$ Target's orientations $(2) \times$ Repetitions (6). The main session consisted of 216 trials in each block-designed condition (432 trials total): Tactile or Auditory conditions $(2) \times$ Target's contrasts $(6) \times$ Target's orientations (2) $\times$ Repetitions (9). The order of these conditions was randomly assigned in each trial and counterbalanced among the participants. The order of the block-designed conditions (tactile/auditory conditions) was also counterbalanced among the participants.

In Exp. 2, we presented the tactile stimulus at either index finger, forearm, or chest in left side of the participants' body as a blocked design (Index finger, Forearm, and Chest conditions). The Without-Touched condition was also included. The visual targets were presented at either left or right side relative to the fixation. Thus, the target and the tactile stimuli were either congruent (Congruent condition) or incongruent (Incongruent condition) in space. After 24 trials of the training session (Target's positions $(2) \times$ Target's orientations $(2) \times$ Repetitions (6)), 80 trials of the main session were introduced in each block-designed condition (240 trials in total): Tactile Conditions (2) $\times$ Target's positions (2) $\times$ Target's orientations $(2) \times$ Repetitions (10). The order of these conditions were randomly assigned in each trial and counterbalanced among the participants. The order of the block-designed conditions (Index finger/Forearm/Chest conditions) was also counterbalanced among the participants.

In Exp. 3, we asked the participants to uncross or cross their hands as a blocked design. While the tactile stimulus was applied to the left or right index finger of each participant, the visual targets were presented on either the left or right side relative to the fixation point for each half of the participants, respectively. Thus, the target and the tactile stimuli were either congruent (Congruent condition) or incongruent (Incongruent condition) in space both in the Hands-Crossed and Hands-Uncrossed conditions. The Without-Touched condition was also included. After 24 trials of the training session (Target's positions $(2) \times$ Target's orientations $(2) \times$ Repetitions $(6)$ ), 80 trials of the main session were introduced in each block-designed condition (160 trials total): Tactile Conditions $(2) \times$ Target's positions $(2) \times$ Target's orientations (2) $\times$ Repetitions (10). The order of these conditions was randomly assigned in each trial and counterbalanced among the participants. The order of the block-designed conditions (Hands-Uncrossed/Crossed conditions) was also counterbalanced among the participants. 

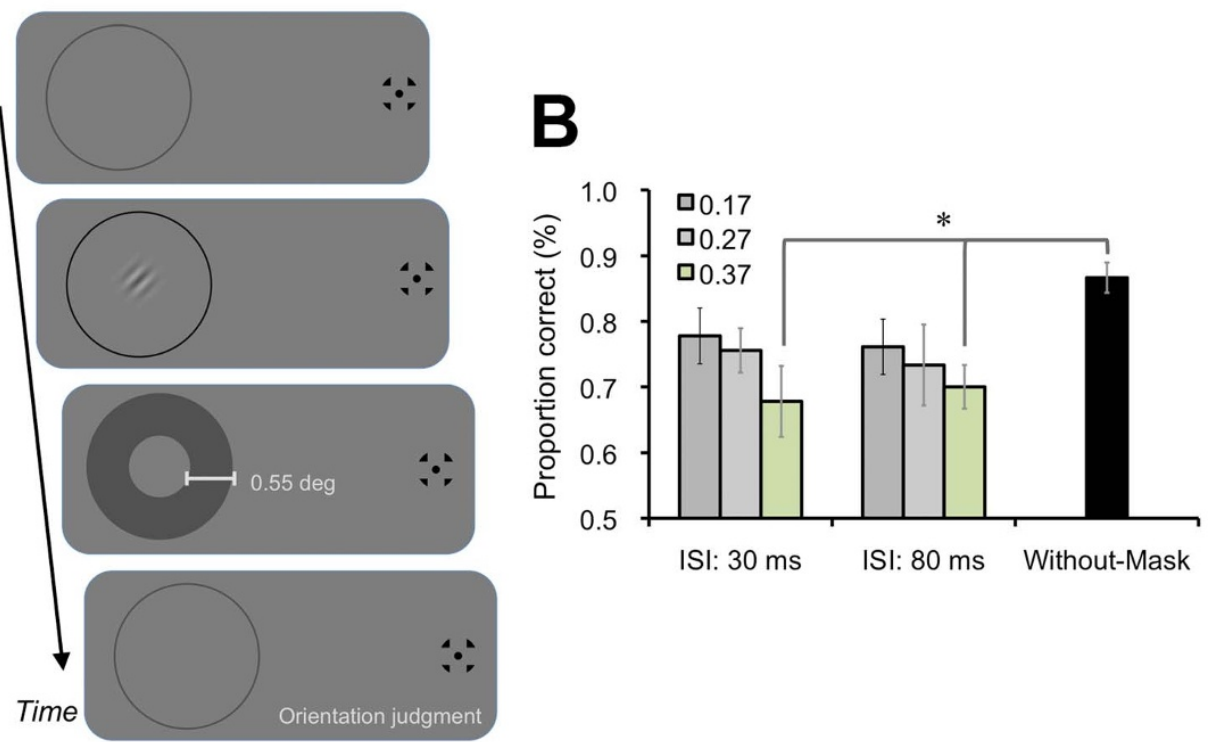

Figure $5 \mid$ Visual metacontrast masking effect. (A) In order to investigate what kind of visual stimuli could induce the suppression effect on visual perception comparable with the tactile suppression effect, we presented a visual masker ( $0.55 \mathrm{deg}$ of width) instead of the tactile stimulus at a surrounding position of the visual target. The contrast of the visual masker was either $0.17,0.27$, or 0.37 (Weber contrast), and the ISI between visual target and masker was $30 \mathrm{~ms}$ or $80 \mathrm{~ms}$. The Without-Mask condition was also included as a baseline. We introduced 240 main session trials (Mask Conditions (2) $\times$ Mask contrasts (3) $\times$ ISIs (2) $\times$ Target's orientations (2) $\times$ Repetitions (10)), followed by 72 training session trials (3 Repetitions). Except for these differences, the stimulus, apparatus, and procedure were identical to those used in Exps. 2-4. (B) Results. The one-way repeated measures ANOVA found a significant main effect of the conditions $[F(6,48)=3.14, p<.05]$. The post hoc test revealed that the proportions of correct responses for visual discrimination in the conditions with 0.37 of contrast and with $30 \mathrm{~ms}$ and $80 \mathrm{~ms}$ of ISI were significantly lower than in the Without-Mask condition $(p<.05)$. Error bars denote the standard error of the mean $(\mathrm{N}=9)$. An asterisk indicates the conditions with a significant difference $(p<.05)$.

In Exp. 4, we manipulated the inter-stimulus interval (ISI) between the visual targets and tactile stimuli from $-250 \mathrm{~ms}$ (tactile stimulus first) to $+250 \mathrm{~ms}$ (visual target first) in 50-ms steps. The tactile stimulus was applied to the left index finger of the participants. We also included the Without-Touched condition. The visual targets were presented on the left side relative to the fixation point. The black ring was presented from the tactile or visual target onset to the tactile or visual target offset. After 72 trials of the training session (visual onset timing relative to the black ring onset $(9) \times$ Target's orientations (2) $\times$ Repetitions (4)), 360 trials of the main session were introduced: ISIs (9) $\times$ Tactile Conditions $(2) \times$ Target's orientations $(2) \times$ Repetitions (10). The order of these conditions was randomized in each trial and counterbalanced among the participants.

1. Breitmeyer, B. G. Visual Masking: An Integrative Approach. New York: Oxford University Press. (1984).

2. Kahneman, D. Method, findings, and theory in studies of visual masking. Psychol. Bull. 70, 404-425 (1968).

3. Enns, J. T. \& Di Lollo, V. What's new in visual masking? Trends. Cogn. Sci. 4, 345-352 (2000)

4. Macknik, S. L. \& Livingstone, M. S. Neuronal correlates of visibility and invisibility in the primate visual system. Nat. Neurosci. 1, 144-149 (1998).

5. Ernst, M. O. \& Bülthoff, H. H. Merging the senses into a robust percept. Trends Cogn. Sci. 8, 162-169 (2004).

6. Stein, B. E., London, N., Wilkinson, L. K. \& Price, D. D. Enhancement of perceived visual intensity by auditory stimuli: A psychophysical analysis. J. Cogn. Neurosci. 8, 497-506 (1996).

7. Meredith, M. A. \& Stein, B. E. Interactions among converging sensory inputs in the superior colliculus. Science 221, 389-391 (1983).

8. Calvert, G. A., Spence, C. \& Stein, B. E. The handbook of multisensory processing. Cambridge: MIT Press (2004).

9. Driver, J. \& Spence, C. Multisensory perception: beyond modularity and convergence. Curr. Biol. 10, R731-R735 (2000).

10. Calvert, G. Crossmodal processing in the human brain: Insights from functional neuroimaging studies. Cereb. Cortex 11, 1110-1123 (2001).

11. Driver, J. \& Noesselt, T. Multisensory interplay reveals crossmodal influences on 'sensory-specific' brain regions, neural responses, and judgments. Neuron 57, 11-23 (2008).

12. Graziano, M. S. \& Gross, C. G. Spatial maps for the control of movement. Curr. Opin. Neurobiol. 8, 195-201 (1998).
13. Driver, J. \& Spence, C. Attention and the crossmodal construction of space. Trends Cogn. Sci. 2, 254-262 (1998).

14. Weinstein, S. Intensive and extensive aspects of tactile sensitivity as a function of body part, sex, and laterality. in The skin senses, eds Kenshalo, D., Springfield, R. \& Thomas, C. pp. 195-222 (1968).

15. Gescheider, G. A. \& Niblette, R. K. Cross-modality masking for touch and hearing. J. Exp. Psychol. 74, 313-320 (1967).

16. Gillmeister, H. \& Eimer, M. Tactile enhancement of auditory detection and perceived loudness. Brain Res. 1160, 58-68 (2007).

17. Soto-Faraco, S., Spence, C., Fairbank, K., Kingstone, A., Hillstrom, A. P. \& Shapiro, K. A crossmodal attentional blink between vision and touch. Psychon. Bull. Rev. 9, 731-738 (2002).

18. Gallace, A., Auvray, M., Tan, H. Z. \& Spence, C. When visual transients impair tactile change detection: a novel case of crossmodal change blindness? Neurosci. Lett. 398, 280-285 (2006).

19. Spence, C. \& Driver, J. Audiovisual links in exogenous covert spatial orienting. Percept. Psychophys. 59, 1-22 (1997).

20. Spence, C. \& Parise, C. Prior-entry: a review. Conscious. Cogn. 19, 364-379 (2010).

21. Botvinick, M. \& Cohen, J. Rubber hands 'feel' touch that eyes see. Nature 391, 756 (1998).

22. Taylor-Clarke, M., Jacobsen, P. \& Haggard, P. Keeping the world a constant size: object constancy in human touch. Nat. Neurosci. 7, 219-220 (2004).

23. Kennett, S., Taylor-Clarke, M. \& Haggard, P. Noninformative vision improves the spatial resolution of touch in humans. Curr. Biol. 11, 1188-1191 (2001).

24. Mirams, L., Poliakoff, E., Brown, R. J. \& Lloyd, D. M. Vision of the body increases interference on the somatic signal detection task. Exp. Brain Res. 202, 787-794 (2010).

25. Rizzolatti, G., Scandolara, C., Matelli, M. \& Gentilucci, M. Afferent properties of periarcuate neurons in macaque monkey. II. Visual responses. Behav. Brain Res. 2, 147-163 (1981).

26. Lloyd, D. M., Shore, D. I., Spence, C. \& Calvert, G. A. Multisensory representation of limb position in human premotor cortex. Nat. Neurosci. 6, 17-18 (2003).

27. Fiorio, M. \& Haggard, P. Viewing the body prepares the brain for touch: effects of TMS over somatosensory cortex. Eur. J. Neurosci. 22, 773-777 (2005).

28. Cardini, F., Longo, M. R. \& Haggard, P. Vision of the body modulates somatosensory intracortical inhibition. Cereb. Cortex 21, 2014-2022 (2011).

29. Ghazanfar, A. A. \& Schroeder, C. E. Is neocortex essentially multisensory? Trends Cogn. Sci. 10, 278-285 (2006) 
30. Duhamel, J. R., Colby, C. L. \& Goldberg, M. E. Ventral intraparietal area of the macaque: congruent visual and somatic response properties. J. Neurophysiol. 79, 126-136 (1998).

31. Avillac, M., Denève, S., Olivier, E., Pouget, A. \& Duhamel, J. R. Reference frames for representing visual and tactile locations in parietal cortex. Nat. Neurosci. 8 , 941-949 (2005).

32. Iriki, A., Tanaka, M. \& Iwamura, Y. Coding of modified body schema during tool use by macaque postcentral neurones. Neuroreport 7, 2325-2330 (1996).

33. Iriki, A., Tanaka, M., Obayashi, S. \& Iwamura, Y. Self-images in the video monitor coded by monkey intraparietal neurons. Neurosci. Res. 40, 163-173 (2001)

34. Alpern, M. Metacontrast. J. Opt. Soc. Am. 43, 648-657 (1953).

35. Francis, G. Cortical dynamics of lateral inhibition: metacontrast masking. Psychol. Rev. 104, 572-594 (1997)

36. Brainard, D. H. The Psychophysics Toolbox. Spat. Vis. 10, 433-436 (1997)

37. Pelli, D. G. The VideoToolbox software for visual psychophysics: transforming numbers into movies. Spat. Vis. 10, 437-442 (1997).

38. Thaler, L., Schütz, A. C., Goodale, M. A. \& Gegenfurtner, K. R. What is the best fixation target? The effect of target shape on stability of fixational eye movements. Vision Res. 76, 31-42 (2013).

\section{Acknowledgments}

We thank Yoich Sugita and Makoto Wada for their comments and suggestions. We also appreciate Shoko Yabuki for providing the illustrations. This research was supported by a Grant-in-Aid for Young Scientists (B) from JSPS (No. 25780446) and the Ministry of Education, Culture, Sports, Science and Technology, Grant-in-Aid for Specially Promoted Research (No. 19001004).

\section{Author contributions}

Conceived and designed the experiments: S.H., I.M. Performed the experiments: I.M. Analyzed the data: I.M., S.H. Wrote the paper: S.H., M.I.

\section{Additional information}

Competing financial interests: The authors declare no competing financial interests.

How to cite this article: Ide, M. \& Hidaka, S. Tactile stimulation can suppress visual perception. Sci. Rep. 3, 3453; DOI:10.1038/srep03453 (2013).

\footnotetext{
(c) (i) $(-)$ This work is licensed under a Creative Commons Attribution-

cc) ${ }_{\mathrm{BY}} \mathrm{NC}$ ND NonCommercial-NoDerivs 3.0 Unported license. To view a copy of this license visit http://creativecommons.org/licenses/by-nc-nd/3.0
} 